\title{
PLAG1, the prototype of the PLAG gene family: Versatility in tumour development (Review)
}

\author{
FREDERIK VAN DYCK, JEROEN DECLERCQ, CAROLINE V. BRAEM and WIM J.M. VAN DE VEN
}

Laboratory for Molecular Oncology and Flanders Interuniversity Institute for Biotechnology,

Department of Human Genetics, K.U. Leuven, Herestraat 49/602, B-3000 Leuven, Belgium

Received October 6, 2006; Accepted November 14, 2006

\begin{abstract}
Recent studies of human tumours as well as genetically engineered mouse tumour models have established the importance and versatility of the PLAG1 oncogene in tumourigenesis. The PLAG1 proto-oncogene was discovered by studying the $\mathrm{t}(3 ; 8)(\mathrm{p} 21 ; \mathrm{q} 12)$ chromosome translocation, which frequently occurs in human pleomorphic adenomas of the salivary glands. PLAG1 encodes a developmentally regulated, SUMOylated and phosphorylated zinc finger transcription factor, recognizes a specific bipartite DNA consensus sequence regulating expression of a spectrum of target genes, and has two structurally related family members, i.e. the PLAGL1 and PLAGL2 gene. Ectopic PLAG1 overexpression, in many cases due to promoter swapping, causes deregulation of expression of a variety of PLAG1 target genes. This was established by microarray analysis, which indicated that the oncogenic capability of PLAG1 is mediated, at least partly, by the IGF-II mitogenic signaling pathway. Oncogenic activation of PLAG1 is also a crucial event in other human tumours, including lipoblastoma, hepatoblastoma, and AML. The oncogenic potential of PLAG1 has been confirmed in in vitro experiments, which also established IGF-II and IGF-IR as key pathway elements, similarly as in many human tumours. Furthermore, generation of conditional PLAG1 transgenic mouse strains revealed tumour development in a variety of targeted tissues, establishing the versatility of the PLAG1 oncogene and pointing towards a window of opportunity for therapeutic intervention studies. In contrast to the pleiotropic oncogenic potential of PLAG1, its family member PLAGL1,
\end{abstract}

Correspondence to: Professor Wim J.M. Van de Ven, Laboratory for Molecular Oncology and Flanders Interuniversity Institute for Biotechnology, Department of Human Genetics, K.U. Leuven, Herestraat 49/602, B-3000 Leuven, Belgium

E-mail:wim.vandeven@med.kuleuven.be

Key words: pleomorphic adenoma gene 1, pleomorphic adenoma gene-like 1, pleomorphic adenoma gene-like 2, tumourigenesis, transcription factor, transgenic, mouse model, insulin-like growth factor II which is localized in an imprinted region on chromosome $6 q 24-25$, is defined by various studies as a tumour-suppressor gene. Finally, the PLAGL2 family member is not only structurally but also functionally more closely related to PLAG1 and has recently also been implicated in AML, both in humans and in genetically modified mice. Collectively, these observations emphasize a more general importance of the PLAGl gene in tumour development. In light of the fact that IGF-IR is implicated in many human tumours, the diversity in PLAG1-induced mouse tumour models, most of which seem to involve Igf 2 signaling, provides useful in vivo platforms to start testing the effects of inhibitors, such as Igf- $1 \mathrm{r}$ inhibitors, on tumour development in distinct tissues or organ types.

\section{Contents}

1. Introduction

2. PLAG1, a crucial oncogene in pleomorphic adenoma of salivary glands

3. Features of the PLAG1 gene transcripts and proteins

4. PLAG1-induced tumours in humans and mice

5. Features of the PLAG1 family members, PLAGL1 and PLAGL2

6. Conclusions

\section{Introduction}

Genetics has played without any doubt a major and successful role in molecular oncology and, after all those years, it is currently still of critical importance; nowadays, especially with respect to develop effective therapeutic intervention protocols. With its focus in the past on the identification and characterization of genes involved in tumour development, genetics has already led to significant contributions to differential diagnosis. In a strategic alliance between various disciplines, relevant genes were identified, for instance, through molecular analysis of cytogenetically well-defined chromosome aberrations. Thereafter, the products of these genes were biochemically characterized and their pathogenetic functions elucidated. This led subsequently to opportunities to consider them in the context of the various types of cancer, with which society is confronted. Such genes appeared to constitute key elements of a variety of more complex cascades and, used as starting 
PLAG1 (8q12)
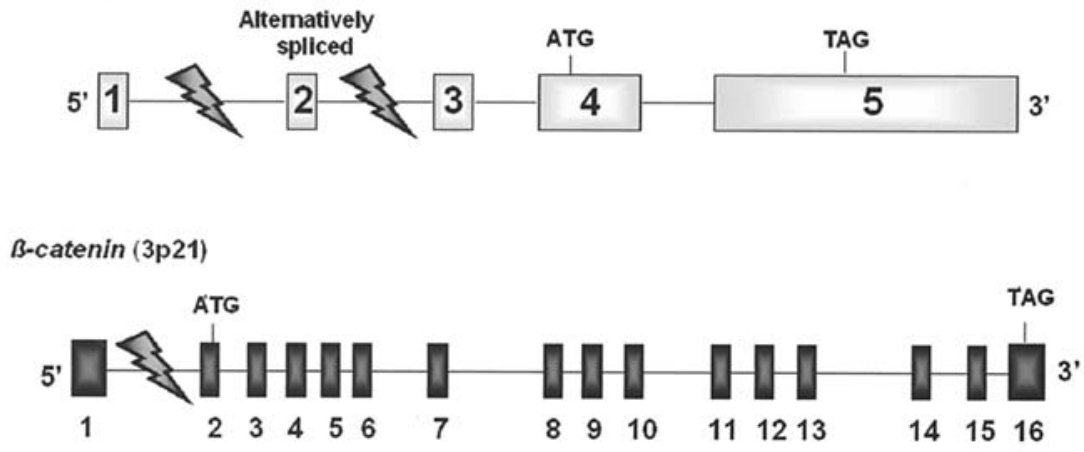

PLAG1/ß-catenin
1
12

B-catenin/PLAG1
\begin{tabular}{|l|l|l|l|}
\hline 3 & 4 & 5 \\
\hline 2 & 3 & 4 & 5 \\
\hline
\end{tabular}

Figure 1. Schematic representation of the nature and origin of $\beta$-catenin/PLAG1 and PLAG1/ $\beta$-catenin fusion transcripts in human pleomorphic adenomas of the salivary glands with $\mathrm{t}(3 ; 8)(\mathrm{p} 21 ; \mathrm{q} 12)$. The chromosomal organization of the PLAG1 gene (light grey boxes) and the $\beta$-catenin gene (black boxes) are given. Positions of translocation breakpoints (arrows), translation initiation sites (ATG) and stop codons (TAG) are indicated. Schematic compositions of 4 hybrid transcripts are presented in the lower part.

points to further unravel these, a better understanding of the complex molecular mechanisms involved in tumourigenesis was obtained. At present, genetics is more focused on the integration of the acquired knowledge in the development of platforms for therapeutic interventions.

In this review, we draw attention to the PLAG gene family, since genetically engineered mouse tumour model systems are expected to be of great instrumental value in studies relating to therapeutic interventions. These tumour model systems have been generated mainly on the basis of the PLAG1 oncogene, which was discovered in studies of pleomorphic adenomas of the salivary glands $(1,2)$. A crucial factor in this is the fact that PLAGl can induce a variety of tumour types in mice and that most of these tumours, if not all, involve the insulin-like growth factor 2 (Igf-2) signalling pathway, which is by many studies also implicated in a wide variety of human tumours $(3,4)$. In this latter perspective, it is evident that human IGF-IR constitutes an important therapeutic target and a subject of intensive research with therapeutic objectives.

\section{PLAG1, a crucial oncogene in pleomorphic adenoma of salivary glands}

Pleomorphic adenomas of the salivary gland have been characterized very well by cytogenetics, with several hundreds of such tumours karyotyped. In addition to a cytogenetic subgroup with a normal karyotype, the largest subgroup of pleomorphic adenomas is characterized by recurrent chromosomal rearrangements, particularly reciprocal translocations, most frequently with a breakpoint at chromosome 8q12. By positional cloning, this affected chromosomal region was characterized and a novel gene, designated pleomorphic adenoma gene 1 (PLAG1), was found to be consistently rearranged (1). In the specific case of $\mathrm{t}(3 ; 8)(\mathrm{p} 21 ; \mathrm{q} 12)$, the
CTNNB1 gene on chromosome $3 \mathrm{p} 21$ is involved as translocation partner. Since CTNNB1 is the chromosome translocation partner of PLAG1 in $25 \%$ of all pleomorphic adenomas of the salivary glands, it is considered its preferential translocation partner. In both genes, the breakpoints are consistently localized between the upstream regulatory promoter sequence and the coding exons. Under normal circumstances, the CTNNB1 gene, encoding the $\beta$-catenin protein involved in cell-cell adhesion and the WG/WNT signalling pathway, is highly and ubiquitously expressed, whereas PLAG1 expression is only readily detectable in foetal tissues. As a result of the translocation, the constitutively active $C T N N B 1$ promoter drives ectopic expression of the otherwise developmentally regulated PLAG1 gene and vice versa. As the coding sequences of both genes are invariably preserved, the molecular mechanism is classified as 'promoter swapping' (Fig. 1).

As an approach to assess the importance of the translocation partners of PLAG1, a second recurrent translocation was characterized, i.e. the $\mathrm{t}(5 ; 8)(\mathrm{p} 13 ; \mathrm{q} 12)$. In these cases, promoter swapping brings the coding sequence of PLAG1 under the control of the ubiquitously expressed gene for the leukemia inhibitory factor receptor (LIFR), a gene structurally unrelated to CTNNB1. Regulation of PLAG1 by the LIFR promoter similarly leads to increased PLAG1 expression (5). Analysis of pleomorphic adenomas without aberrant karyotypes revealed cryptic rearrangements of the PLAGl gene, leading to fusions between PLAG1 and CTNNB1, SII/TCEA1, or, as recently described, $C H C H D 7(6,7)$. Similar to the CTNNB1-PLAG1 and LIFR-PLAG1 fusions, these all result in overexpression of the PLAGl gene.

In summary, aberrant activation or ectopic overexpression of PLAG1 is a crucial pathogenetic event in pleomorphic adenomas of the salivary glands, occurring more often than originally suggested by cytogenetics. 


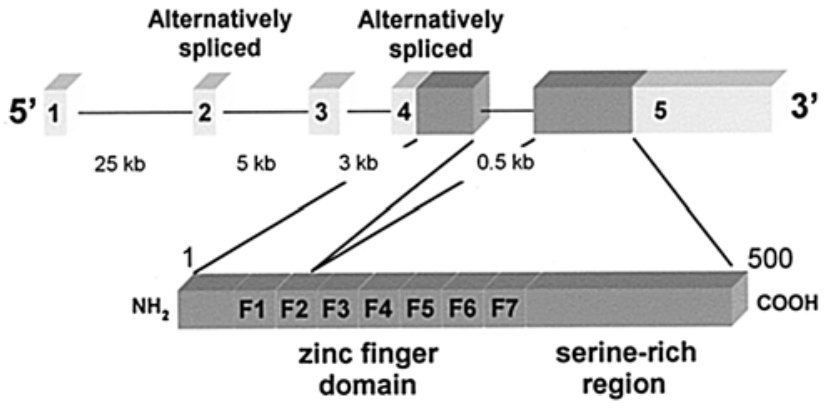

Figure 2. Schematic representation of human PLAG1 gene and protein. The exon/intron distribution of the PLAG1 gene is shown at the top. Exons 4 and 5 contain the coding sequences (dark grey). The deduced PLAG1 protein, depicted in the lower part, contains 7 zinc fingers $(\mathrm{F} 1-\mathrm{F} 7)$ of the $\mathrm{C}_{2} \mathrm{H}_{2}$ type and a $\mathrm{COOH}$-terminal serine-rich region.

\section{Features of the PLAG1 gene transcripts and proteins}

The human PLAG1 gene on chromosome 8q12 consists of 5 exons of which only the last part of exon 4 and the first part of exon 5 (1500 bp in total) are coding. The predominant transcript is $7.3 \mathrm{~kb}$ and three alternatively spliced transcripts of 7.2, 7.0 and $6.9 \mathrm{~kb}$ have been reported (8). Translation of the major transcript results in a protein with a deduced molecular weight of $56 \mathrm{kDa}(1)$. Due to loss of exon 4 by alternative splicing, an $\mathrm{NH}_{2}$-terminal truncated PLAG1 isoform of $47 \mathrm{kDa}$ has been described (Fig. 2). Seven successive $\mathrm{C}_{2} \mathrm{H}_{2}$ zinc finger domains, responsible for DNA binding and possibly for nuclear targeting of the protein, are confined to the $\mathrm{NH}_{2}$-terminal half of the protein, followed by a serine-rich $\mathrm{COOH}$-terminus. The latter domain exhibits transcriptional activation capacity (9). The major PLAG1 protein undergoes post-translational modification such as SUMOylation, acetylation and phosphorylation (see below).

Expression studies have revealed that PLAG1 is developmentally regulated. PLAGl is mainly expressed as a $7.3 \mathrm{~kb}$ transcript, detectable in different foetal human tissues including lung, liver and kidney. In adult tissues, including heart, brain, placenta, lung, liver, skeletal muscle, kidney and pancreas, expression of PLAG1 remains below Northern blot detection levels (1). Expression in adult murine tissues, including intestine, tongue, brain, kidney, liver, lung, skeletal muscle, spleen, stomach, thymus, prostate, salivary gland and uterus, is likewise undetectable, with the exception of testis, ovary and heart (10).

PLAG1 is a genuine transcription factor. Several studies have identified and characterized PLAG1 as a genuine transcription factor (9). The most profound characteristics of such a transcription factor are its ability to localize to the nucleus, to target a recognition motif in the DNA and to activate or repress transcription of downstream target genes from its binding site, due to its inherent transactivation capacity. The presence of 7 canonical $\mathrm{C}_{2} \mathrm{H}_{2}$ zinc fingers in PLAG1, a motif that has been shown to have a predominant role in protein-DNA recognition (11), and the transactivation capacities of the $\mathrm{COOH}$-terminal domain of PLAG1 (9) suggest that the PLAG1 protein may indeed act as a transcriptional regulator.
Nuclear localization of PLAG1. PLAG1 is a nuclear protein, as established in various studies. For instance, exogenous PLAG1, overexpressed in COS-1 kidney fibroblast cells, is localized in the nucleus, as demonstrated by immunofluorescence studies (12). The same intracellular distribution can be seen when PLAG1-GFP fusion proteins are expressed in 293 cells. Studies on cultured human pleomorphic adenoma cells of the salivary gland with PLAG1-specific antibodies shows nuclear localization of the endogenous PLAG1 protein (13). Although passive diffusion could drive PLAG1 through the nuclear pores, because of its low molecular weight, two regions of the PLAG1 protein have been shown to be independently relevant for its nuclear localization. One is the region with the zinc finger domains in the $\mathrm{NH}_{2}$-terminal half of the protein. On the other hand, two potential nuclear localization signals (KRKR and KPKR) reside in the $\mathrm{NH}_{2}$ terminal region, of which only the first one is functional by serving as a karyopherin $\alpha 2$ recognition site (13). Karyopherin $\alpha 2$, a member of the importin family, was shown to be a PLAG1-interacting protein. Importin $\beta$ docks the karyopherincargo complex to the nuclear pore, followed by a translocation of this complex through the pore in an energy-dependent way.

PLAG1 specifically recognizes a bipartite DNA-binding consensus sequence. The presence of seven canonical zinc fingers in PLAG1 suggests that the protein has DNA-binding potential. PLAG1 specifically recognizes and transiently activates transcription from a bipartite DNA-binding consensus sequence, consisting of a core sequence (GRGGC) and a G-cluster (RGGK), separated by 6-8 random nucleotides. Two non-contiguous regions in PLAG1 are essential for this binding, fingers 6 and 7 interacting with the core and finger 3 recognizing the G-cluster (12).

Identification of IGF-II as a bona fide PLAG1 target gene. A computer search in the eukaryotic promoter database (EPD) (14) with the PLAG1 binding consensus sequence (GRGGC (N)7 RGGK) revealed potential PLAG1 recognition motifs in the promoter region of many genes. Because of its role in tumourigenesis, initial attention was drawn to the human insulin-like growth factor II (IGF-II) gene, especially to promoter 3. Five potential PLAG1 binding sites were predicted therein. Reporter experiments confirmed functionality since $I G F-I I$ expression can be highly stimulated by PLAG1. Moreover, a drastic up-regulation of $I G F-I I$ promoter 3 transcripts coincides with PLAG1 activation in human pleomorphic adenomas of the salivary glands, indicating a correlation between PLAGI and IGF-II expression (12). Since IGF-II is overexpressed in several types of human neoplasias, potently stimulating cell proliferation through autocrine or paracrine mechanisms $(15,16)$, these results suggest that the predicted oncogenic capacity of PLAG1 is mediated, at least partly, by activating the IGF-II mitogenic pathway.

Microarray screening for target genes of PLAG1. The discovery of $I G F-I I$ as one of PLAG1's bona fide target genes, provided the first clue to understanding the pathogenetic mechanism according to which PLAG1 exerts its oncogenic potential (12). The identification via microarray analysis and subsequent validation of other PLAG1 target 

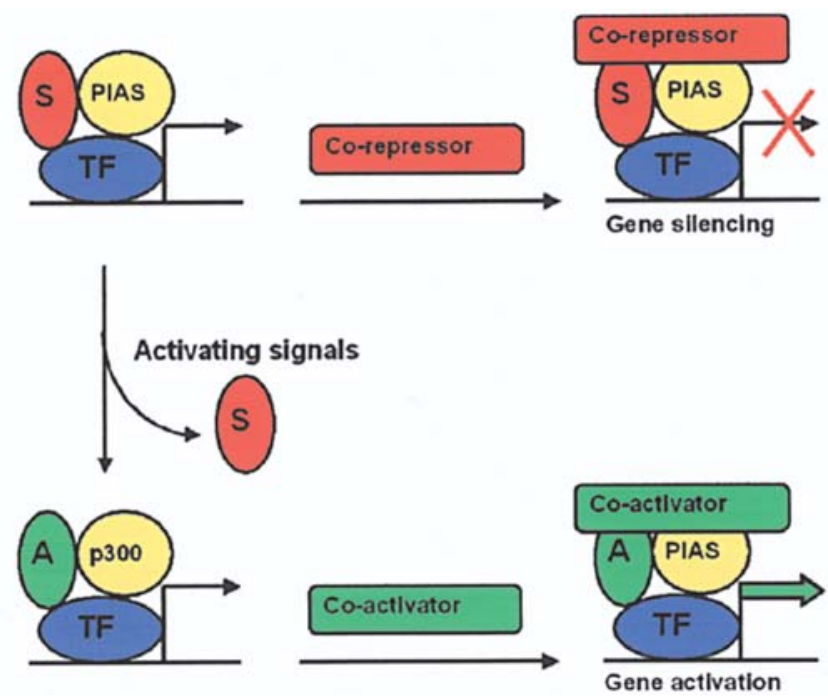

Figure 3. Activation of a transcription factor: a dynamic interplay between SUMOylation and acetylation. A transcription factor (TF), in a repressed state resulting from SUMOylation involving E3-ligases, such as PIAS, can respond to activating signals by deSUMOylation and subsequent acetylation, for example by p300. Acetylation occurs at the same lysine residues as SUMOylation and consequently eradicates the function of the repressive domain to which co-repressors are recruited if SUMOylated. Moreover, it enhances transcriptional activation by recruiting other coactivators. TF, transcription factor; S, SUMO; A; acetylation.

genes have provided further clues as to the underlying mechanisms involved in PLAG1-induced oncogenesis (17). In in vitro transformation studies, expression of 47 genes were consistently found to be up-regulated by PLAG1, whereas 12 genes were repressed. One of the largest classes of up-regulated targets consists of growth factors, including insulin-like growth factor 2, cytokine-like factor-1, bonederived growth factor, vascular endothelial growth factor and placental growth factor. Of interest is the observed and validated up-regulation of genes of two distinct imprinted gene clusters, i.e. Igf2/H19 and Dlk1/Gtl2. In silico studies supported the notion that the PLAG1-induced genes represent direct PLAG1 targets. The in vitro results have also been compared to in vivo expression profiling data obtained in studies in which profiles in PLAG1-induced pleomorphic adenomas of the salivary glands were compared to those in normal salivary glands (17). This yielded a set of 12 consistently induced genes with in silico support for direct PLAG1 targets. In contrast, none of genes that were found down-regulated in the in vitro setting appeared to have their expression significantly altered in the in vivo setting.

It could be postulated that one of the roles of PLAG1 in human tumour formation is to influence cell proliferation via induction of growth factors, such as IGF-II. On the other hand, the expression profile reveals a possible role of PLAG1 in other cellular processes. For instance, the observed upregulated levels of vascular endothelial growth factor (VEGF), placental growth factor (PIGF) and Ephrin B1 seem to link PLAG1 to vasculogenesis and/or angiogenesis, which is also important in the context of tumour development.

Dynamic interplay between SUMOylation and acetylation of PLAG1. Generally, transcription factors modulate gene
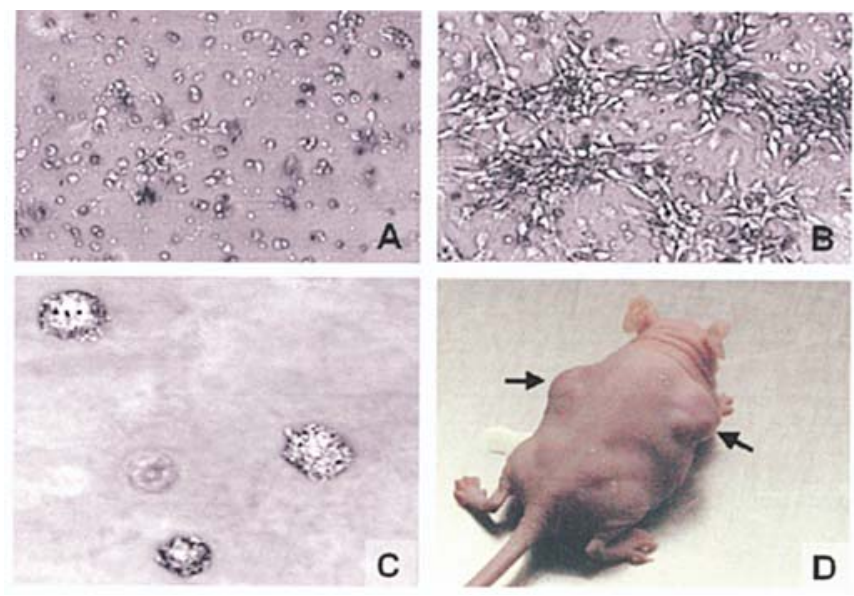

Figure 4. Overexpressed PLAG1 proteins are able to transform NIH-3T3 cells. The in vitro transforming capacity of PLAG1 was studied in retroviral transduction studies using NIH-3T3 cells and focus forming and for soft agar assay systems. (A) Control NIH-3T3 cells; (B) Focus formation of PLAG1 overexpressing NIH-3T3 cells; (C) Colony formation in soft agar of PLAG1 overexpressing NIH-3T3 cells; (D) Tumour formation upon subcutaneous injection of PLAG1 overexpressing NIH-3T3 cells into both flanks of athymic nude mice. The relative positions of the resulting tumours are indicated by arrows. [Figure adapted from Hensen et al (41)].

expression in a molecular context of a protein complex involving interaction partners. Numerous studies have also pointed towards the importance of post-translational modifications of transcription factors, such as phosphorylation, acetylation, ubiquitination, and SUMOylation, in achieving a dynamic regulation of gene expression $(18,19)$.

From yeast two-hybrid assays, confirmed by GST pulldown experiments, it appeared that all members of the PLAG protein family interact with several members of the PIAS protein family. These have been shown to play a role as an E3ligase in the SUMOylation of a variety of proteins, including several transcription factors (20-23). The SUMOylation process, which consists in the covalent and reversible conjugation of Small Ubiquitin-related modifiers to target proteins, has recently been reviewed extensively (reviewed in refs. 24-28). With respect to PLAG1, studies have clearly established that PLAG1 is SUMOylated at more than one lysine residue and that SUMOylation has an apparent inhibitory effect on the transcriptional capacity of PLAG1 $(23,29)$. This is consistent with the notion that modification of proteins with SUMO is more and more recognized as an important regulatory process in a diverse set of cellular pathways. It is well established that it might influence the stability, the sub-nuclear localisation and, in case of transcription factors, the transcriptional capacity of target proteins $(24,30,31)$.

Although the precise physiological impact of this posttranslational modification on the functioning of these transcription factors remains to be established, it is tempting to speculate that SUMOylation might play a regulatory role in the dissociation of the transcriptional complexes of which PLAG1 is an integral part (23). Moreover, PLAG1 and PLAGL2 are also modified by acetylation, targeting the same lysine residues and resulting in an opposite effect on their 
transactivation capacity (29). Since SUMO itself does not have repression activity, a model emerges whereby the different functional consequences of acetylation and SUMOylation could be the result of a change in affinity for different interacting protein partners, functioning as coactivators and co-repressors, respectively (Fig. 3).

Phosphorylation of PLAG1. First indications came from simple experiments with radio-labelled phosphoric acid and ${ }^{32} \mathrm{P}$ incorporation in PLAG1, which revealed phosphorylation of PLAG1. Moreover, ${ }^{32} \mathrm{P}$-incorporation was stronger if okadaic acid, a serine/threonine phosphatase inhibitor, was added to the labelling medium, whereas addition of the protein tyrosine phosphatase inhibitor, sodium vanadate, exerted no effect on ${ }^{32} \mathrm{P}$-incorporation. Okadaic acid is a highly selective inhibitor of the serine/threonine protein phosphatase type 1 (PP1) and 2A (PP2A) (32). Since okadaic acid caused increased PLAG1 phosphorylation, it was concluded that PLAG1 is phosphorylated on serine and/or threonine residues and its de-phosphorylation is, at least partly, regulated by PP1 and/ or PP2A. Further studies indicated that PLAG1 is mainly phosphorylated in the $\mathrm{COOH}$-terminal serine-rich transactivation domain. The presence of phosphorylation sites in the transactivating domain of PLAG1 raises the possibility that phosphorylation plays a role in regulating its transactivating potential (Braem C.V., PhD thesis, K.U. Leuven).

\section{PLAG1-induced tumours in humans and mice}

Studies have indicated that PLAG1 is involved in various human tumours and can induce a variety of tumours in PLAG1 transgenic mice, as outlined below in more detail.

With respect to human tumours, PLAG1 is consistently rearranged, not only in a large subgroup of pleomorphic adenomas of the salivary glands (1,5-7), as discussed above, but also in lipoblastomas $(33,34)$. Lipoblastomas are benign paediatric neoplasias resulting from transformation of adipocytes. They typically contain adipose cells in different stages of maturation, primitive mesenchymal cells, myxoid matrix, and fibrous trabeculae. Clonal chromosomal rearrangements involving the $8 \mathrm{q} 11-13$ region are present in $\sim 70 \%$ of lipoblastomas (33-35). The way in which PLAG1 is turned on, i.e. by promoter swapping, is comparable between pleomorphic adenoma of the salivary glands and lipoblastoma, however, the fusion partners for PLAG1 differ. In lipoblastoma, currently known PLAG1 swapping partners are the gene encoding hyaluronic acid synthase 2 (HAS2) on chromosome band $8 \mathrm{q} 24$ and collagen $1 \alpha 2$ (COL1A2) on chromosome band 7q22. Each provides PLAG1 with a strong promoter after chromosomal rearrangement (34).

Recent studies have implicated the PLAG1 gene in hepatoblastoma. These studies started from the evidence that $8 \mathrm{q}$ amplification is associated with poor prognosis in hepatoblastoma. The PLAG1 oncogene appeared highly expressed in 19 out of 20 tumours as compared to normal liver tissue. Furthermore, qRT-PCR revealed that the expression level of PLAG1 was 3-12 times greater in hepatoblastoma tumours and cell lines compared to the levels in age-matched normal livers. Moreover, the levels appeared comparable to that in foetal liver tissue. Using luciferase reporter assays, it was demonstrated that, also in hepatoblastoma-derived cell lines, PLAG1 transactivates transcription from the embryonic IGF-II promoter P3. Of interest in this context is that up-regulation of IGF-II was observed in almost all hepatoblastomas (36).

Other studies have shown that PLAG1 also seems to be implicated in leukaemia. PLAGl expression is increased in $20 \%$ of human acute myeloid leukaemia (AML) samples, that contain in most cases the $C B F B-M Y H 11$ fusion gene, coding for the fusion protein $\mathrm{CBF}$-SMMHC, due to chromosome 16 inversion, inv(16)(p13;q22) (37). This fusion protein inhibits the core binding factor (CBF), resulting in a block of haematopoietic differentiation (38). However, $\mathrm{Cbfb}^{+/ C b f b-M Y H 11}$ chimeric mice do not develop any malignancies in their first year of life, but they do show a strong predisposition for AML, as shown after injection with the DNA alkylating mutagen, $\mathrm{N}$-ethyl-N-nitrosourea (39). Thus, the class-II mutation (inducing impairment of haematopoietic differentiation) Cbfb-MYH11 contributes to leukaemic transformation, but additional genetic events are definitely required. Retroviral promoter insertion studies with the $C b f b^{+/ C b f b-M Y H 11}$ chimeras, identified Plagl as a candidate gene for Cbfb-MYH11associated leukaemogenesis in the mouse (40). Up-regulation of Plag1, a class-I mutation (conferring haematopoietic progenitor expansion, such as increase in proliferation, cell survival, or self-renewal potential), induces AML in cooperation with Cbfß-SMMHC. However, PLAG1 induces only moderate $I g f 2$ transcript levels in bone marrow cells, which are not maintained in leukaemic samples. This suggests that Plag1 may affect an Igf2-independent pathway in leukaemogenesis or that it plays a role in the initiation but not the maintenance of leukaemia (37).

PLAG1 ectopic expression has also been observed in uterine leiomyoma, leiomyosarcomas and in smooth muscle tumours $(6,34)$.

Oncogenic capacity of PLAG1 in vitro. To establish the oncogenic capacity of PLAG1 in vitro classic transfection experiments were performed using NIH-3T3 cells. NIH-3T3 cells overexpressing PLAG1 display the typical hallmarks of neoplastic transformation: the cells a) lose cell-cell contact inhibition, b) show anchorage-independent growth, c) partially abrogate the serum requirement for the growth of the NIH-3T3 cells, and d) are able to induce tumours upon injection in nude mice (Fig. 4) (41). These in vitro transformation data are consistent with the assumed oncogenic role of PLAG1 described for the human tumours, discussed above. Importantly, among the multiple genes, Igf-2 was consistently the strongest up-regulated upon transduction of cells with PLAG1 DNA, indicating that the IGF-1R signalling pathway might be crucial. This up-regulation is most likely not a cell type-dependent effect since a similar up-regulation of IGF-II was achieved by inducing PLAG1 expression in a human epithelial kidney cell line (293 cells). Altogether, the oncogenic capacity of PLAG1 is thus mediated, at least partly, by activating the IGF-II mitogenic pathway (41). We further hypothesized that the transforming effect of IGF-II, observed in our model system, is thus mainly mediated via IGF-IR, activation of which is known to cause increased mitogenesis, primarily via the MAPK signalling pathway (42). This was undisputably confirmed in later studies using IGF-IR-negative 

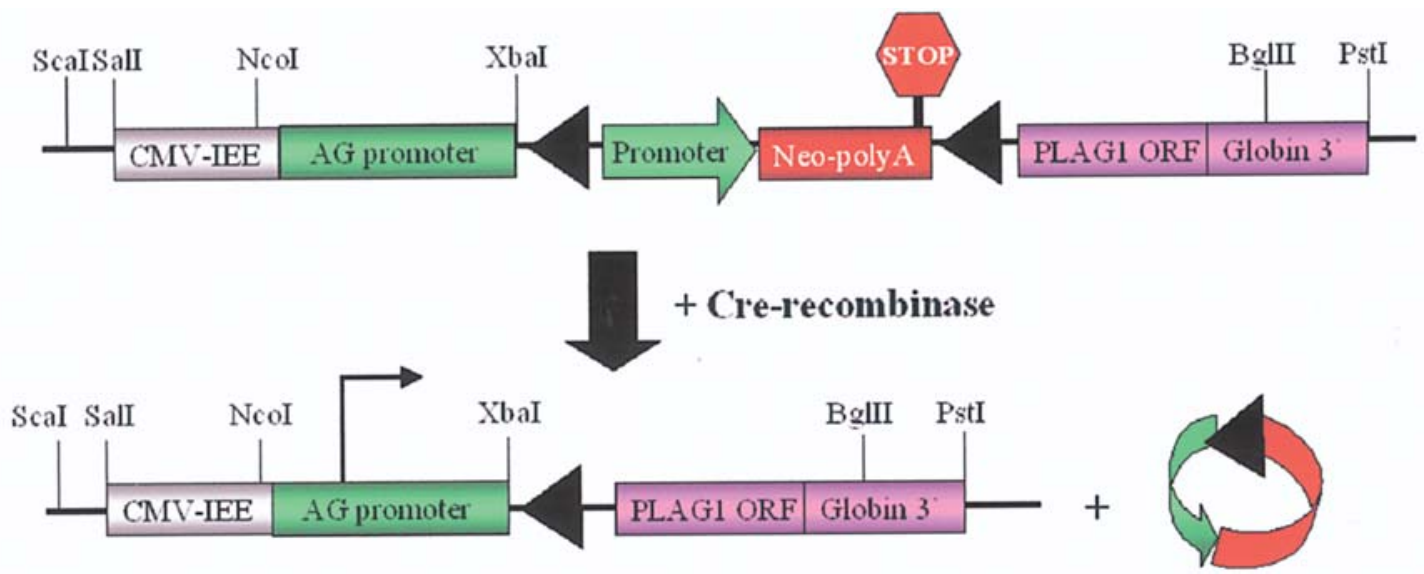

Figure 5. Schematic representation of the PLAG1 transgene used in the generation of PLAG1 transgenic mouse strains. Human PLAG1 cDNA, containing the complete open reading frame for PLAG1 and sequences encoding a haemagglutinin tag at the 5' end of the PLAG1 cDNA (indicated in the figure as 'PLAG1 ORF'), was cloned in the pCAGGS vector, bringing its expression under control of the CMV-IEE-AG promoter. A stop-cassette was inserted between the AG promoter and the PLAG1 coding sequences, consisting of the Neo gene (Neo-polyA), under expression control of the PGK promoter (green arrow) and flanked on both sides by loxP sites (black triangles) (upper half). A Cre-mediated intramolecular recombination event leads to excision and circularisation of the floxed neomycin cassette. As a result, PLAG1 is placed under the control of the CMV-IEE-AG promoter (lower half). CMV-IEE, CMV immediate early enhancer; AG, chicken $ß$-actin/rabbit B-globin hybrid promoter; $\mathrm{pA}$, polyadenylation.
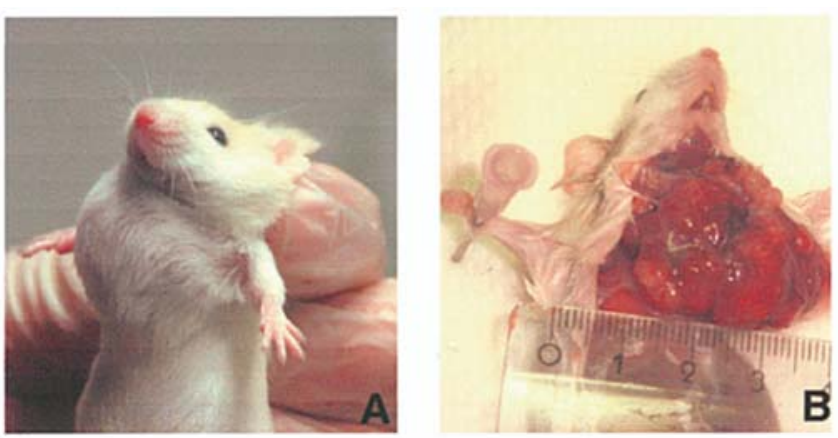

Figure 6. Pleomorphic adenoma development in PLAG1 overexpressing salivary glands of transgenic mice. (A) Large salivary gland tumour mass in a 5-week-old MMTV-LTR-Cre ${ }^{+/-} / \mathrm{PLAG}^{+/-}$mouse (P1-Mcre). (B) Macroscopic appearance at necropsy of the tumour mass of the 5-week-old P1-Mcre mouse shown in (A).

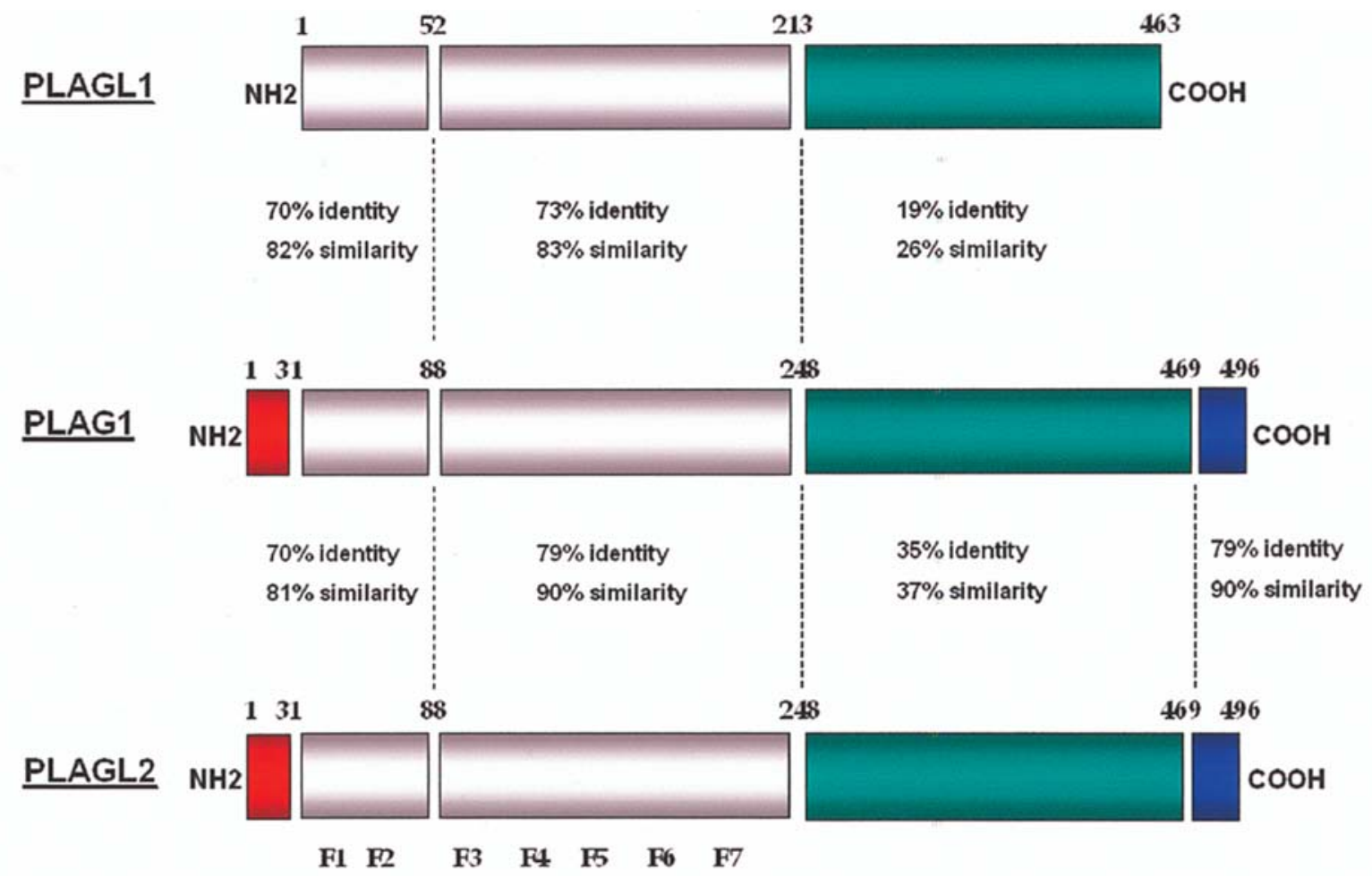

Figure 7. The PLAG family of zinc finger proteins. A schematic comparison is given between the architecture of the PLAG zinc finger proteins. The identity/similarity between different parts of the proteins is indicated [adapted from Kas et al (9)]. 
cells (R-cells) (43), which could not be transformed by PLAG1 anymore (41).

Oncogenic capacity of PLAG1 in vivo. To explore the role of PLAG1 in neoplastic transformation in vivo in the context of a complex organism, transgenic mouse strains were developed $(2,44)$. The most versatile strains are conditional transgenics in which activation of overexpression of the PLAG1 transgene as well as the tissue distribution of such overexpression can be manipulated (e.g., by Cre-mediated activation and targeted expression, respectively) (Fig. 5) (2). This approach could lead to the elucidation of a pathogenetic role of PLAG1 in the affected tissue and would provide the means to identify the direct target genes of PLAG1 that form the downstream part of the molecular signal transduction cascade involved in the observed pathology (45). Targeting of PLAG1 overexpression to the salivary (using MMTV-Cre) as well as the mammary glands (using MMTV-Cre or Wap-Cre) led to the development of tumours in these organs. In the salivary glands of the offspring, huge tumours developed within about five weeks in $100 \%$ of the animals. They were classified as pleomorphic adenomas and share many histopathologic features with their human counterparts (Fig. 6). In some of the older mice, malignant characteristics could be observed, consistent with the fact that $2-17 \%$ of human pleomorphic adenomas, although benign at first, can progress to malignancy after all ('carcinoma ex-pleomorphic adenoma') (46-48). All tumours expressed a variety of PLAG1 target genes, including those from the two independent imprinted gene clusters ( $I g f 2$, $H 19, D l k 1$, and Gtl2); especially $I g f 2$ at very high levels. Complete inactivation of the Igf2 gene in such offspring led, on the average, to a noticeable delay in tumour appearance of 2 -fold (Declercq J, unpublished data). This together with in vitro data discussed above suggests that IGF-IR could be critical in PLAG1-induced tumours. Expression of PLAG1 is also being targeted to other organs and preliminary results indicate that the oncogenic impact of PLAG1 in those transgenic mouse strains is not limited to salivary and mammary glands (Van Dyck F and Declercq J, unpublished data). Evaluation via expression profiling of the observed lesions often reveals expression of PLAG1 target genes, invariably Igf2 at high levels. The possibility to obtain other PLAG1-induced lesions enables studying various PLAG1induced tumour types. Of utmost importance in this context is to establish to what extent the various tumours in these mice mimic particular human tumours.

In conclusion, the PLAG1 proto-oncogene seems to exert its oncogenic potential via cellular signalling triggered by IGF-II and its cognate receptor IGF-IR, at least partially. Generally, this pathway is initiated via ligand binding which leads to receptor dimerization, autophosphorylation, and subsequent activation of downstream substrates, resulting in activation of the MAPK signalling pathway, primarily responsible for mitogenesis, and the anti-apoptotic and proliferative PI-3 kinase/Akt pathway (4). The ability of IGF-IR signalling to affect these two major pathways in tumourigenesis has contributed to the wide interest in finding agents to block IGF-IR signalling $(49,50)$, not least from a clinical perspective.

\section{Features of the PLAG1 family members, PLAGL1 and PLAGL2}

PLAG1, together with the two structurally related proteins PLAGL1 (PLAG-like 1; also called LOT1 or ZAC1) and PLAGL2 (PLAG-like 2), constitute a small subfamily of zinc finger proteins (9). The three PLAG proteins are mainly homologous in their $\mathrm{NH}_{2}$-terminal zinc finger domain $(73 \%$ and $79 \%$ identity to PLAG1 for PLAGL1 and PLAGL2, respectively), whereas the $\mathrm{COOH}$-terminal region is more divergent (Fig. 7).

PLAGL1, an imprinted cell cycle control gene. Although PLAGL1 shows high homology to PLAG1 in the DNAbinding domain, the DNA-binding specificities of these two proteins seem to differ slightly. The consensus binding site for PLAGL1, GGGGGGCCCC, does not include the G-cluster, the second part of the bipartite consensus sequence identified for PLAG1 and localized 7 nucleotides downstream the core GGRGGCC (41). PLAGL1 is maternally imprinted, such as several genes implicated in growth control, which suggests a dosage-dependent function $(51,52)$. Human PLAGL1/ZAC (Zinc finger that regulates apoptosis and cell cycle arrest) localizes to the chromosomal imprinted region 6q24-25 linked to growth inhibition and deleted in many solid tumours (53), and shares the ability with p53 to inhibit growth of tumour cells by controlling apoptosis and cell cycle progression (54). Likewise, expression of Lot1 (Lost on transformation 1), the rat orthologue of PLAGL1, is lost during spontaneous transformation of ovary surface epithelial cells in vitro (55). Moreover, hypermethylation of chromosomal region 6q24-25 leading to transcriptional silencing of PLAGL1 has been reported in a variety of human cancers, including ovarian cancer (56). The gene can thus be classified as a tumoursuppressor and is targeted by HDAC1, a transcriptional regulator that was shown to affect expression of several other genes with proposed tumour suppressor activity, such as JunB, Apc2, metallothionein 1, metallothionein 2, Prss11 (57). Furthermore, the zinc finger domain of Zac links DNA binding to HAT signalling, since coordinated binding of Zac to p300 regulates HAT activity by simultaneously increasing substrate affinities and catalytic activity (58).

Consistent with a role for PLAGL1 in development and its ability to induce the gene for the type 1 receptor for PACAP, implicated in glucose-stimulated insulin release, overexpression of PLAGL1 in humans, resulting from a loss of maternal imprinting or duplication of the active paternal allele, is associated with intra-uterine growth restriction and transient neonatal diabetes mellitus (59-62). The human isoform PLAGL1 is widely expressed, both in foetal and adult tissues $(9,53)$. In adults, Zac shows a high expression in most steroidresponsive tissues where it potently co-activates or co-represses the hormone-dependent activity of nuclear receptors, implicated in regulation of cell growth, differentiation, homeostasis, and development in a cell-specific context (58).

A different expression pattern is found in rodents. The mouse homologue is found to be highly expressed in the pituitary gland, whereas the rat homologue is expressed in a limited number of normal rat tissues, including ovary, pancreas, testis and uterus (63). 
PLAGL2, a developmentally regulated PLAG1-like protooncogene. Structurally and functionally, PLAG1 and PLAGL2 are the most similar. PLAG1 and PLAGL2 have similar DNA-binding affinity, different from that of PLAGL1. This is reflected in the ability to induce common target genes, such as the positive regulation of the IGF-II gene. In contrast to PLAGL1, PlagL2 was also shown to be SUMOylated and acetylated (29). PLAG1 and PlagL2 have similar oncogenic properties in vitro, since aberrant expression of PlagL2 makes fibroblasts able to grow independent of serum, unaffected by the inability to anchor and by the contact with neighbouring cells (41). Furthermore, PLAGL2 has recently also been associated with human cancer, since retroviral promoter insertion studies with the $C b f b^{+/ C b f b-M Y H 11}$ chimeras identified both Plag1 and PlagL2 activation as essential genetic events in leukaemogenesis, and PLAGL2 expression is preferentially induced in human AML samples with inv(16). Moreover, PLAGL2 might even participate in AML development in cooperation with other fusion genes, since its levels were also significantly higher in a panel of $t(8 ; 21)$ and $t(15 ; 17)$ AMLsamples tested (37). These findings are in contradiction with the identification of PlagL2 as an iron depletion- or hypoxiainducible gene that eventually drives cells into apoptosis. In transient transfection experiments, PLAGL2 is able to enhance transcription from an HRE (hypoxia-inducible factor-1 response element) containing LDHA (lactate dehydrogenase A) promoter. Furthermore, PLAGL2 and HIF-1 synergistically activate transcription of the LDHA promoter in such experiments (64). In addition, expression of PlagL2 in the nucleus of desferrioxamine-treated cells coincided with expression of the pro-apoptotic factor Nip-3, which contains a hypoxia-responsive element that is activated by PLAGL2, independent of HIF-1 (65). Therefore, PlagL2 was shown to function as a tumour suppressor protein in association with HIF-1 in specific conditions of hypoxia or iron depletion.

PLAGL2 is also a developmentally regulated gene based on its expression pattern. Similar to PLAG1, PLAGL2 transcripts are present in a variety of foetal human tissues whereas its levels are undetectable by Northern blot in adult tissues (9). The expression pattern of PlagL2 in adult mouse tissues is even more distinct from the situation in human than that of Plag1. PlagL2 is ubiquitously present in adult mouse tissues, including heart, brain, lung, spleen, liver, skeletal muscle, kidney and testis (64). The marked differences in the range of expression of the PLAG genes, between human and rodents, are intriguing but remain unexplained. Recently, expression patterns of the three genes were compared, focussing on the central nervous system, peripheral nervous system, and the sensory and neuroendocrine lineages. Similarly, while the three members of the Plag gene family are co-expressed in some of these lineages, they also display unique and sometimes complementary patterns of expression in other tissues (59).

To get a better understanding of the role of PlagL2 during mouse development, a transgenic mouse strain was developed in which PlagL2 was fully disrupted by replacing its second coding exon with the LacZ reporter gene (Braem, C.V., PhD thesis, K.U. Leuven). PlagL2 $2^{-/}$mouse pups are only mildly reduced in body weight and size at birth. However, by the end of the first week, both male and female PlagL2 ${ }^{-/}$mice are on average $65 \%$ smaller than control littermates. This severe early postnatal growth retardation of PlagL2 knockout pups coincides with an extreme high lethality (Braem C.V., PhD thesis, K.U. Leuven, 2004).

\section{Conclusions}

In light of the above and looking to the future, it is increasingly evident that proteomics will have a major impact on PLAG1 research, since it provides the opportunity to look at the biological model systems, that have been generated, as a whole rather than at individualized parts. The capacity to acquire quickly the vast amount of data on biological molecules has greatly advanced science. Proteomics, along with genomics, transcriptomics, and metabolomics will certainly contribute in a major way to the scientific ventures ahead in this particular field. In general, the impact of proteomics in basic and clinical research is just starting to blossom, and particularly in the field of biomedical research, proteomics is expected to have a huge impact both in the discovery of new diagnostics and therapeutics. For these innovative reasons, one should put proteomics at the core of future research efforts using the generated PLAG1-induced tumour model systems. In this manner, optimal capitalization might be expected from the variety of unique PLAGl-induced mouse tumour model systems, which are very well suited to collect a wealth of data using proteomics. Special attention should be given to IGF-IR-mediated tumourigenesis, since IGR-IR is involved in many human tumours. Therefore, the generated data might have an impact relevant to many human tumours in case the data from the mouse model systems can be translated accordingly, and likely provide avenues for early diagnosis.

Finally, the generated PLAG1 model systems could be instrumental in studies of various aspects of IGF-II signalling-mediated tumourigenesis, including early diagnosis and future therapeutic interventions, which upon translation to human tumours would be beneficial to mankind.

\section{Acknowledgements}

This study was supported in part by the 'Geconcerteerde Onderzoeksactie (GOA-010, 2002-2006)', the 'Fonds voor Wetenschappelijk Onderzoek Vlaanderen (FWO, G.0099.02, 2002-2005)', the Foundation for Biochemical and Pharmaceutical Research and Education, and the 'Fortis Bank (FB) Verzekeringen-programma voor Kankeronderzoek'. 'Dit werk is ook tot stand gekomen dankzij een wetenschappelijke onderzoeksbeurs van' the Belgian Federation against Cancer, non-profit organization. F. Van Dyck has been awarded a bursary by the 'Instituut voor de Aanmoediging van Innovatie door Wetenschap en Technologie in Vlaanderen (IWTVlaanderen)'. C. Braem and J. Declercq were involved in this research as Aspirants of the FWO.

\section{References}

1. Kas K, Voz ML, Roijer E, Astrom AK, Meyen E, Stenman G and Van de Ven WJ: Promoter swapping between the genes for a novel zinc finger protein and beta-catenin in pleiomorphic adenomas with $\mathrm{t}(3 ; 8)(\mathrm{p} 21 ; \mathrm{q} 12)$ translocations. Nat Genet 15 : 170-174, 1997. 
2. Declercq J, Van Dyck F, Braem CV, Van Valckenborgh IC, Voz M, Wassef M, Schoonjans L, Van Damme B, Fiette L and Van de Ven WJ: Salivary gland tumors in transgenic mice with targeted PLAG1 proto-oncogene overexpression. Cancer Res 65: 4544-4553, 2005.

3. LeRoith D and Roberts CT Jr: The insulin-like growth factor system and cancer. Cancer Lett 195: 127-137, 2003.

4. Samani AA, Yakar S, Leroith D and Brodt P: The role of the IGF system in cancer growth and metastasis: overview and recent insights. Endocr Rev (In press).

5. Voz ML, Astrom AK, Kas K, Mark J, Stenman G and Van de Ven WJ: The recurrent translocation $t(5 ; 8)(p 13 ; q 12)$ in pleomorphic adenomas results in upregulation of PLAG1 gene expression under control of the LIFR promoter. Oncogene 16: 1409-1416, 1998

6. Astrom AK, Voz ML, Kas K, Roijer E, Wedell B, Mandahl N, Van de Ven W, Mark J and Stenman G: Conserved mechanism of PLAG1 activation in salivary gland tumors with and without chromosome 8q12 abnormalities: identification of SII as a new fusion partner gene. Cancer Res 59: 918-923, 1999.

7. Asp J, Persson F, Kost-Alimova M and Stenman G: CHCHD7PLAG1 and TCEA1-PLAG1 gene fusions resulting from cryptic, intrachromosomal $8 \mathrm{q}$ rearrangements in pleomorphic salivary gland adenomas. Genes Chromosomes Cancer 45: 820-828, 2006.

8. Queimado L, Lopes C, Du F, Martins C, Bowcock AM, Soares J and Lovett M: Pleomorphic adenoma gene 1 is expressed in cultured benign and malignant salivary gland tumor cells. Lab Invest 79: 583-589, 1999.

9. Kas K, Voz ML, Hensen K, Meyen E and Van de Ven WJ: Transcriptional activation capacity of the novel PLAG family of zinc finger proteins. J Biol Chem 273: 23026-23032, 1998.

10. Hensen K, Braem C, Declercq J, Van Dyck F, Dewerchin M, Fiette L, Denef C and Van de Ven WJ: Targeted disruption of the murine Plag1 proto-oncogene causes growth retardation and reduced fertility. Dev Growth Differ 46: 459-470, 2004.

11. Wolfe SA, Nekludova $\mathrm{L}$ and Pabo $\mathrm{CO}$ : DNA recognition by Cys2His 2 zinc finger proteins. Annu Rev Biophys Biomol Struct 29: 183-212, 2000.

12. Voz ML, Agten NS, Van de Ven WJ and Kas K: PLAG1, the main translocation target in pleomorphic adenoma of the salivary glands, is a positive regulator of IGF-II. Cancer Res 60: 106-113, 2000.

13. Braem CV, Kas K, Meyen E, Debiec-Rychter M, Van De Ven WJ and Voz ML: Identification of a karyopherin alpha 2 recognition site in PLAG1, which functions as a nuclear localization signal. J Biol Chem 277: 19673-19678, 2002.

14. Cavin Perier R, Junier T and Bucher P: The Eukaryotic Promoter Database EPD. Nucleic Acids Res 26: 353-357, 1998.

15. Khandwala HM, McCutcheon IE, Flyvbjerg A and Friend KE: The effects of insulin-like growth factors on tumorigenesis and neoplastic growth. Endocr Rev 21: 215-244, 2000.

16. Daughaday WH: The possible autocrine/paracrine and endocrine roles of insulin-like growth factors of human tumors. Endocrinology 127: 1-4, 1990.

17. Voz ML, Mathys J, Hensen K, Pendeville H, Van Valckenborgh I, Van Huffel C, Chavez M, Van Damme B, De Moor B, Moreau Y and Van de Ven WJ: Microarray screening for target genes of the proto-oncogene PLAG1. Oncogene 23: 179-191, 2004.

18. Gill G: Something about SUMO inhibits transcription. Curr Opin Genet Dev 15: 536-541, 2005.

19. Lee PS, Chang C, Liu D and Derynck R: Sumoylation of Smad4, the common Smad mediator of transforming growth factor-\{beta\} family signaling. J Biol Chem 278: 27853-27863, 2003.

20. Jackson PK: A new RING for SUMO: wrestling transcriptional responses into nuclear bodies with PIAS family E3 SUMO ligases. Genes Dev 15: 3053-3058, 2001

21. Kotaja N, Karvonen U, Janne OA and Palvimo JJ: PIAS proteins modulate transcription factors by functioning as SUMO-1 ligases. Mol Cell Biol 22: 5222-5234, 2002.

22. Sharrocks AD: PIAS proteins and transcriptional regulation more than just SUMO E3 ligases? Genes Dev 20: 754-758, 2006.

23. Van Dyck F, Delvaux ELD, Van de Ven WJM and Chavez MV: Repression of the transactivating capacity of the oncoprotein PLAG1 by SUMOylation. J Biol Chem 279: 36121-36131, 2004.

24. Seeler JS and Dejean A: Nuclear and unclear functions of SUMO. Nat Rev Mol Cell Biol 4: 690-699, 2003.
25. Melchior F, Schergaut M and Pichler A: SUMO: ligases, isopeptidases and nuclear pores. Trends Biochem Sci 28: 612-618, 2003.

26. Verger A, Perdomo J and Crossley M: Modification with SUMO. EMBO Rep 4: 137-142, 2003.

27. Bossis G and Melchior F: SUMO: regulating the regulator. Cell Div 1: 13, 2006.

28. Hay RT: SUMO: a history of modification. Mol Cell 18: 1-12, 2005.

29. Zheng G and Yang YC: Sumoylation and acetylation play opposite roles in the transactivation of PLAG1 and PLAGL2. J Biol Chem 280: 40773-40781, 2005.

30. Melchior F: SUMO - nonclassical ubiquitin. Annu Rev Cell Dev Biol 16: 591-626, 2000.

31. Muller S, Hoege C, Pyrowolakis G and Jentsch S: SUMO, ubiquitin's mysterious cousin. Nat Rev Mol Cell Biol 2: 202-210, 2001

32. Walsh AH, Cheng A and Honkanen RE: Fostriecin, an antitumor antibiotic with inhibitory activity against serine/threonine protein phosphatases types 1 (PP1) and 2A (PP2A), is highly selective for PP2A. FEBS Lett 416: 230-234, 1997.

33. Astrom A, D'Amore ES, Sainati L, Panarello C, Morerio C, Mark J and Stenman G: Evidence of involvement of the PLAG1 gene in lipoblastomas. Int J Oncol 16: 1107-1110, 2000.

34. Hibbard MK, Kozakewich HP, Dal Cin P, Sciot R, Tan X, Xiao S and Fletcher JA: PLAG1 fusion oncogenes in lipoblastoma. Cancer Res 60: 4869-4872, 2000.

35. Bourelle S, Viehweger E, Launay F, Quilichini B, Bouvier C, Hagemeijer A, Jouve JL and Bollini G: Lipoblastoma and lipoblastomatosis. J Pediatr Orthop B 15: 356-361, 2006

36. Zatkova A, Rouillard JM, Hartmann W, Lamb BJ, Kuick R, Eckart M, von Schweinitz D, Koch A, Fonatsch C, Pietsch T, Hanash SM and Wimmer K: Amplification and overexpression of the IGF2 regulator PLAG1 in hepatoblastoma. Genes Chromosomes Cancer 39: 126-137, 2004.

37. Landrette SF, Kuo YH, Hensen K, Barjesteh van Waalwijk van Doorn-Khosrovani S, Perrat PN, Van de Ven WJ, Delwel R and Castilla LH: Plag1 and Plag12 are oncogenes that induce acute myeloid leukemia in cooperation with Cbfb-MYH11. Blood 105: 2900-2907, 2005.

38. Castilla LH, Wijmenga C, Wang Q, Stacy T, Speck NA, Eckhaus M, Marin-Padilla M, Collins FS, Wynshaw-Boris A and Liu PP: Failure of embryonic hematopoiesis and lethal hemorrhages in mouse embryos heterozygous for a knocked-in leukemia gene CBFB-MYH11. Cell 87: 687-696, 1996.

39. Castilla LH, Garrett L, Adya N, Orlic D, Dutra A, Anderson S, Owens J, Eckhaus M, Bodine D and Liu PP: The fusion gene Cbfb-MYH11 blocks myeloid differentiation and predisposes mice to acute myelomonocytic leukaemia. Nat Genet 23: 144-146, 1999.

40. Castilla LH, Perrat P, Martinez NJ, Landrette SF, Keys R, Oikemus S, Flanegan J, Heilman S, Garrett L, Dutra A, Anderson S, Pihan GA, Wolff L and Liu PP: Identification of genes that synergize with Cbfb-MYH11 in the pathogenesis of acute myeloid leukemia. Proc Natl Acad Sci USA 101: 4924-4929, 2004.

41. Hensen K, Van Valckenborgh IC, Kas K, Van de Ven WJ and Voz ML: The tumorigenic diversity of the three PLAG family members is associated with different DNA binding capacities. Cancer Res 62: 1510-1517, 2002.

42. LeRoith D, Werner H, Neuenschwander S, Kalebic T and Helman LJ: The role of the insulin-like growth factor-I receptor in cancer. Ann NY Acad Sci 766: 402-408, 1995

43. Sell C, Dumenil G, Deveaud C, Miura M, Coppola D, De Angelis T, Rubin R, Efstratiadis A and Baserga R: Effect of a null mutation of the insulin-like growth factor I receptor gene on growth and transformation of mouse embryo fibroblasts. Mol Cell Biol 14: 3604-3612, 1994.

44. Zhao X, Ren W, Yang W, Wang Y, Kong H, Wang L, Yan L, $\mathrm{Xu} \mathrm{G}$, Fei J, Fu J, Zhang $\mathrm{C}$ and Wang Z: Wnt pathway is involved in pleomorphic adenomas induced by overexpression of PLAG1 in transgenic mice. Int J Cancer 118: 643-648, 2006

45. Jaisser F: Inducible gene expression and gene modification in transgenic mice. J Am Soc Nephrol 11 (Suppl 16): S95-S100, 2000 .

46. Mizui T, Ishimaru JI, Miyamoto K and Toida M: Malignant transformation of a gigantic pleomorphic adenoma of the submandibular gland: a case report. J Oral Maxillofac Surg 58: $1422-1424,2000$ 
47. Olsen KD and Lewis JE: Carcinoma ex-pleomorphic adenoma: a clinicopathologic review. Head Neck 23: 705-712, 2001.

48. Roijer E, Nordkvist A, Strom AK, Ryd W, Behrendt M, Bullerdiek J, Mark J and Stenman G: Translocation, deletion/ amplification, and expression of HMGIC and MDM2 in a carcinoma ex-pleomorphic adenoma. Am J Pathol 160: 433-440, 2002.

49. Haluska P, Carboni JM, Loegering DA, Lee FY, Wittman M, Saulnier MG, Frennesson DB, Kalli KR, Conover CA, Attar RM, Kaufmann SH, Gottardis M and Erlichman C: In vitro and in vivo antitumor effects of the dual insulin-like growth factor-I/insulin receptor inhibitor, BMS-554417. Cancer Res 66: 362-371, 2006.

50. Wittman M, Carboni J, Attar R, Balasubramanian B, Balimane P, Brassil P, Beaulieu F, Chang C, Clarke W, Dell J, Eummer J, Frennesson D, Gottardis M, Greer A, Hansel S, Hurlburt W, Jacobson B, Krishnananthan S, Lee FY, Li A, Lin TA, Liu P, Ouellet C, Sang X, Saulnier MG, Stoffan K, Sun Y, Velaparthi U, Wong H, Yang Z, Zimmermann K, Zoeckler M and Vyas D: Discovery of a (1H-benzoimidazol-2-yl)-1H-pyridin-2-one (BMS-536924) inhibitor of insulin-like growth factor I receptor kinase with in vivo antitumor activity. J Med Chem 48: 5639-5643, 2005.

51. Piras G, El Kharroubi A, Kozlov S, Escalante-Alcalde D, Hernandez L, Copeland NG, Gilbert DJ, Jenkins NA and Stewart CL: Zac1 (Lot1), a potential tumor suppressor gene, and the gene for epsilon-sarcoglycan are maternally imprinted genes: identification by a subtractive screen of novel uniparental fibroblast lines. Mol Cell Biol 20: 3308-3315, 2000.

52. Smith RJ, Arnaud P, Konfortova G, Dean WL, Beechey CV and Kelsey G: The mouse Zac1 locus: basis for imprinting and comparison with human ZAC. Gene 292: 101-112, 2002.

53. Varrault A, Ciani E, Apiou F, Bilanges B, Hoffmann A, Pantaloni C, Bockaert J, Spengler D and Journot L: hZAC encodes a zinc finger protein with antiproliferative properties and maps to a chromosomal region frequently lost in cancer. Proc Natl Acad Sci USA 95: 8835-8840, 1998

54. Spengler D, Villalba M, Hoffmann A, Pantaloni C, Houssami S, Bockaert J and Journot L: Regulation of apoptosis and cell cycle arrest by Zac1, a novel zinc finger protein expressed in the pituitary gland and the brain. EMBO J 16: 2814-2825, 1997.

55. Abdollahi A, Godwin AK, Miller PD, Getts LA, Schultz DC, Taguchi T, Testa JR and Hamilton TC: Identification of a gene containing zinc-finger motifs based on lost expression in malignantly transformed rat ovarian surface epithelial cells. Cancer Res 57: 2029-2034, 1997.
56. Arima T, Yamasaki K, John RM, Kato K, Sakumi K, Nakabeppu Y, Wake $\mathrm{N}$ and Kono T: The human HYMAI/ PLAGL1 differentially methylated region acts as an imprint control region in mice. Genomics 88: 650-658, 2006.

57. Zupkovitz G, Tischler J, Posch M, Sadzak I, Ramsauer K, Egger G, Grausenburger R, Schweifer N, Chiocca S, Decker T and Seiser C: Negative and positive regulation of gene expression by mouse histone deacetylase 1. Mol Cell Biol 26: 7913-7928, 2006.

58. Hoffmann A, Barz T and Spengler D: Multitasking $\mathrm{C} 2 \mathrm{H} 2$ zinc fingers link Zac DNA binding to coordinated regulation of p300histone acetyltransferase activity. Mol Cell Biol 26: 5544-5557, 2006.

59. Alam S, Zinyk D, Ma L and Schuurmans C: Members of the Plag gene family are expressed in complementary and overlapping regions in the developing murine nervous system. Dev Dyn 234: 772-782, 2005.

60. Gloyn AL, Mackay DJ, Weedon MN, McCarthy MI, Walker M, Hitman G, Knight BA, Owen KR, Hattersley AT and Frayling TM: Assessment of the role of common genetic variation in the transient neonatal diabetes mellitus (TNDM) region in type 2 diabetes: a comparative genomic and tagging single nucleotide polymorphism approach. Diabetes 55: 2272-2276, 2006.

61. Ciani E, Hoffmann A, Schmidt P, Journot L and Spengler D Induction of the PAC1-R (PACAP-type I receptor) gene by p53 and Zac. Brain Res Mol Brain Res 69: 290-294, 1999.

62. Kamiya M, Judson H, Okazaki Y, Kusakabe M, Muramatsu M, Takada S, Takagi N, Arima T, Wake N, Kamimura K, Satomura K, Hermann R, Bonthron DT and Hayashizaki Y: The cell cycle control gene ZAC/PLAGL1 is imprinted - a strong candidate gene for transient neonatal diabetes. Hum Mol Genet 9: 453-460, 2000

63. Huang SM and Stallcup MR: Mouse Zac1, a transcriptional coactivator and repressor for nuclear receptors. Mol Cell Biol 20: $1855-1867,2000$.

64. Furukawa T, Adachi Y, Fujisawa J, Kambe T, Yamaguchi-Iwai Y, Sasaki R, Kuwahara J, Ikehara S, Tokunaga R and Taketani S: Involvement of PLAGL2 in activation of iron deficient- and hypoxia-induced gene expression in mouse cell lines. Oncogene 20: 4718-4727, 2001

65. Mizutani A, Furukawa T, Adachi Y, Ikehara S and Taketani S: A zinc-finger protein, PLAGL2, induces the expression of a proapoptotic protein Nip3, leading to cellular apoptosis. J Biol Chem 277: 15851-15858, 2002. 